\title{
Promover la
}

toma de decisiones

sobre los alumnos

de diseño

Resumen. La toma de decisiones es el proceso mediante el cual se escoge una alternativa sobre otras. Existen decisiones triviales como ¿qué desayunar hoy?, hasta otras de mayor impacto y análisis como escoger la estrategia que asegurará llegar a las metas de ventas.

El diseño no es ajeno a este proceso; muy por el contrario el diseño plantea por definición, una toma de decisión en esencia.Ante la pregunta ¿qué hace a un diseñador, un buen diseñador? destaco por encima de todo, la capacidad de brindar una solución a un problema, lo que significa en el fondo, saber tomar una decisión.

Esta idea invita a ver el proceso de diseño y el proceso creativo como una serie de decisiones relacionadas entre sí, una tras de otra, permitiendo superar los obstáculos del camino y avanzar hacia un resultado esperado.

Por esta razón, es fundamental desarrollar en los alumnos la toma de decisiones durante los proyectos de diseño, empoderándolos para que de forma responsable y seguros de sí mismos, puedan saber discernir entre las alternativas y escoger la mejor opción. Esto los preparará para desempeñarse exitosamente como profesionales y los hará capaces de afrontar todo tipo de retos.

Introducción. Uno de los logros esperados de un estu-

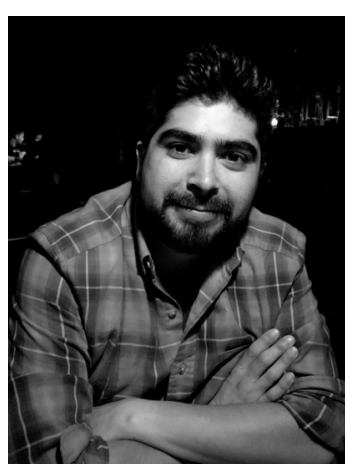

Mtro.Alonso Celis Castro Universidad San Ignacio de Loyola alonsocelis@gmail.com Lima, Perú. 
diante de diseño, es el aprendizaje y ejercicio del proceso de diseño. Este proceso es muy importante ya que a través de él, los diseñadores pueden llevar a cabo proyectos creativos sin morir en el intento y con una probabilidad de éxito mayor que la que ofrece la tan esperada inspiración, la suerte o incluso el "talento artístico" al que aspiran algunos.

Es decir, todo proceso de diseño implica la generación de ideas y la exploración de escenarios. En cada uno de estos componentes, existe un mundo imaginativo donde las ideas van y vienen sin ninguna estructura necesaria. Pero para cuando llegamos al momento de la formulación de propuestas, esas ideas deben de articularse en soluciones que se puedan presentar de una forma racional y lógica.

Por lo tanto, el rol que juega el proceso de diseño es fundamental ya que permite a los diseñadores alcanzar el ¡Eureka! de una forma más segura pero al mismo tiempo mantener una serie de filtros que garantizan que se está yendo por buen camino.

Dicho en otras palabras, el proceso de diseño, al ser una serie de pasos ordenados, les aporta a los diseñadores un modelo de dirección y un esquema de trabajo dividido en fragmentos o etapas, que lo vuelve muy útil y conveniente para logra un resultado más eficiente y eficaz.

Sin embargo, saber los beneficios del proceso de diseño no garantiza a ningún alumno convertirse en buen diseñador. Bruno Munari lo graficaba muy bien en su libro "Diseño y Comunicación Visual”:“...un joven quiere diseñar enseguida un proyecto, de la misma manera que quiere enseguida conducir un coche o tocar un instrumento".

De ahí que explicar qué es y para qué sirve es insuficiente para el propósito de los estudiantes, ya que su verdadero valor se aprecia en la práctica y en el ejercicio de decenas si no son cientos de proyectos en los que el alumno pueda acostumbrarse a pensar conforme al proceso, aprender a equivocarse sin miedo, pero sobre todo, a tomar las decisiones de diseño correctas.

El nuevo mundo donde se diseña. El Iphone, Alexa, Facebook, AirBnB, Uber son ejemplos de un nuevo mundo, uno donde se diferencia entre consumidores tradicionales y consumidores digitales, entre comunicación offline y online; es como dos tipos de seres humanos distintos a los que el diseño debe servir en simultáneo. El impacto de estas nuevas empresas en la vida de las personas es enorme, y a la vez, sorprendente para haber pasado en tan poco tiempo. Esto se debe a diferentes factores que están trasformando la vida como la conocemos: la manera de comunicarnos, de trabajar, de trasportarnos, de aprender, viajar, todo.

La nube, la IA y el machine learning, los smartphones, la omnicanalidad, la nanotecnología, el internet de las cosas, el Big Data, las energías ecoamigables y renovables, la robótica, la integración biológica-digital,las impresoras 3D, entre otros son factores que están cambiando al mundo.

Ya en el 2016, el Foro Económico Mundial anunció: "El mundo ha entrado en lo que ya es considerada la revolución más grande de la historia de la humanidad": La Cuarta Revolución Industrial... ... en la que las empresas, las economías, las sociedades y la política se están transformando de manera esencial."

Si bien este nuevo mundo sugiere una realidad futurista, apalancada en la tecnología, de la misma forma que una película de ciencia ficción; existe también, una necesidad latente de cambio en la sociedad nunca visto antes. El plástico, el calentamiento global,la reducción de las áreas verdes, la pobreza, son ejemplos de problemas sociales y ecológicos que influyen en las personas volviéndolas demandantes de una nueva estructura de las cosas.

En su conjunto, tanto la aceleración de la velocidad del cambio (lo que significaba ser rápido antes, hoy es ser lento), la influencia de la tecnología y el internet en la vida de las personas, así como la preocupación por un planeta sostenible, han empujado a las empresas a una posición en donde para seguir contando con la aceptación de sus consumidores y mantenerse competitivas, deben de cambiar.

Entonces, esto podría explicar la urgente necesidad de innovar de las empresas, ya sea en sus productos, el modelo de servicio, el modelo de negocio e incluso su estructura jerárquica, hay una carrera por el título de empresa innovadora.

Este ímpetu por innovar tiene un lado negativo tanto para las empresas como para los diseñadores, ya que solo por el hecho de hacerlo, sin contar con un objetivo claro, se puede terminar en un proceso ciego a su propósito y 
resumiéndose a la pretensión de que una app, una publicidad en redes sociales o un código QR son soluciones de diseño que resolverán todos sus problemas.

¿Y cómo el diseño sobrevive a este nuevo mundo automatizado, tecnológico, acelerado y demandante?

Astrid Stavro, diseñadora gráfica italiana y directora de Pentagram, menciona:"En esencia,la profesión (de diseñador) no ha cambiado, el proceso de diseño es el mismo. La diferencia es que ahora disponemos de mejores herramientas y de una tecnología que nos permiten plantear y visualizar soluciones más complejas con más rapidez".

Efectivamente, los diseñadores seguimos afrontando los proyectos implementando el mismo proceso de diseño; sin embargo, existen páginas web que automatizan la creación de logotipos, de señaléticas, de íconos; existe galerías enteras (de paga y gratuitas) de webs, sistemas visuales de comunicación corporativa, editores y animadores de videos listos para que coloques el logo de la empresa y puedas descargarlo.

En sí mismo la profesión del diseñador no ha cambiado; sin embargo, se puede decir que el rol del diseñador se ha revalorado apalancado sobre una necesidad de innovación, pero al mismo tiempo se ha deshumanizado, al punto que es solo un proceso de diseño, una serie de pasos a seguir sin ninguna aportación del diseñador (que más asume el rol de ejecutor).

Es increíble como el pensamiento de diseñador, o más conocido como Design Thinking, han encontrado un recibimiento y un valor altísimo en áreas de la empresa, como lo son operaciones, negocios, RRHH, sistemas, marketing, etc.; sin embargo, en las empresas de diseño propiamente, los presupuestos y cronogramas ajustados, empujan a los directores a automatizar la creatividad.

Mi nombre es procesoy vengo de diseño. Es difícil proyectar un proceso de diseño único que pueda aplicarse a todo proyecto de diseño, ya que las características propias del proyecto podrían sugerir un tratamiento especial. Además, existen diferentes diseñadores que gestionan sus proyectos de diferentes maneras.

Sin embargo, en relación con las etapas esenciales, es increíble la similitud que comparten diferentes diseñadores y personas que trabajan en industrias creativas. (Figura 1)
Estas etapas pueden sintetizarse en el siguiente cuadro:

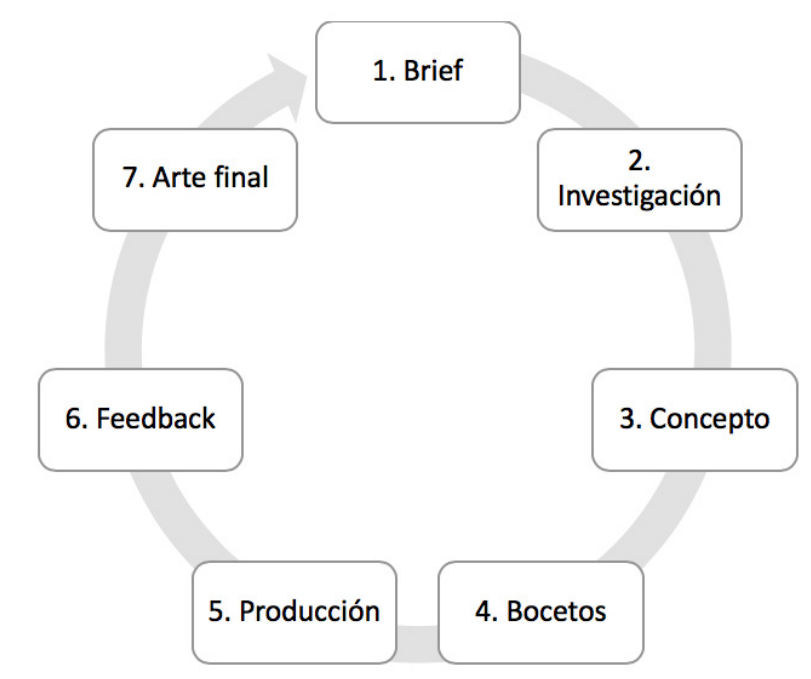

Fig. 1 (Gráfico de las etapas esenciales del proceso de diseño)

1. Brief.- El reconocido diseñador argentino Ronald Shakespear dice que un buen diseñador es aquel que tiene una oreja grande para escuchar a la gente. Es por eso que todo proceso empieza con el cliente, escuchando, preguntando y anotando el problema a solucionar.

El diseñador debe analizar esta información, validar la problemática (o reformularla) y calcular el alcance de las acciones que podría tomar solucionarlo. El resultado de este primer paso se puede ver en el brief de diseño, con los objetivos a alcanzar escritos de forma clara y sencilla.

2. Investigación.- Existen 4 tipos de investigación que se deben de hacer en forma paralela:

- Investigación informativa: Con ella se debe profundizar en todo lo relacionado al cliente (su misión, visión, sus valores), sus productos o servicios, la problemática a resolver. Asimismo se debe investigar a la competencia $y$ al mercado.

- Investigación del público objetivo: El propósito del diseño son las personas, y su misión, la de mejorar sus vidas. Por esta razón no se podrá tener ningún buen resultado de diseño si no se conoce al público al que se desea alcanzar. En la medida de lo posible se debe profundizar en las motivaciones de consumo, en los agentes influenciadores y en el grado de satisfacción para descubrir insights interesantes que puedan ayudar en el diseño. 
- Investigación gráfica: Consiste en una búsqueda de referencias importantes que puedan influir e inspirar nuevas formas en la etapa del boceto y producción del diseño.

- Investigación creativa: Ayuda a marca el estándar de solución a la cual aspiramos llegar con nuestro diseño. Esta investigación no siempre se relaciona a los objetivos de diseño del brief, más por el contrario, es una expectativa del nivel creativo al que deseamos llegar.

3. Conceptualización.- En esta etapa, es donde se da el la lluvia de ideas, las cuales se articulan para engendrar el concepto. Este se convertirá en el norte del proyecto ayudando a tomar las decisiones de diseño y marcando los límites creativos.

4. Bocetos o prototipos.- Una vez conceptualizado el diseño, se pasa a una de las etapas más activas y divertidas del proceso del diseño. La acción de "bocetear" implica un número de ensayos y errores, avances y retrocesos, de crear y destruir para otra vez volver a crear. La experiencia y el conocimiento se vuelven relativos frente a la capacidad de abstracción y pensamiento lateral propio de los diseñadores para llegar a propuestas realmente originales.

5. Producción del diseño.- Validados los bocetos, se puede pasar a la etapa de producción donde de forma sofisticada, el diseño se arma de acuerdo a los bocetos. Esta etapa es más técnica pero además altamente creativa ya que se busca obtener la mejor calidad con los recursos que se encuentran a disposición. El resultado deberá responder a un trabajo minucioso y altamente detallista.

6. Arte final.- Como último paso, se testea el diseño y se pide feedback de distintos frentes, para realizar los últimos ajustes y poder desarrollar el arte final.

Como se mencionó antes, estos representan las etapas comunes que en su esencia, están presentes en todos los modelos del proceso de diseño. Algunos incluyen una etapa de seguimiento y medición, otros juntan la etapa del brief con la investigación, son finalmente matices.

Aprender vs practicar. Aprender el proceso de diseño no es difícil, practicarlo tampoco, pero sí exige un grado mínimo de disciplina para poder pasar por todas las etapas, experimentarlas y sentir como se va adquiriendo una mayor capacidad (tanto intelectual como creativa) para responder a la problemática.
Es muy fácil caer en la tentación de sub estimar a la teoría del proceso de diseño, creer que de tan obvia que es, no es necesaria y solo sirve a manera de checklist. En muchas clases, los alumnos no investigan al cliente por lo tanto empiezan el proceso sin saber los objetivos que deben cumplir. Otros, no investigan a la competencia, y no es hasta el final en que descubren que su idea no tiene nada de original, ya que ha sido usada por otras empresas. Un caso muy repetitivo, es el quedarse con la primera idea, lo que conlleva a un concepto pobre y poco atractivo.

Estos errores ocurren también cuando no se tiene costumbre de implementar el proceso de diseño; en las clases, me he encontrado con casos de alumnos que dibujan muy bien, realizan bocetos que parecen obras de arte; sin embargo, cuando le pido que me expliquen el proceso de cómo llegaron a esa idea o cómo es que se alcanzó tal solución, se quedan callados, no por timidez, sino porque no lo saben, solo apelaron al "talento artístico"y en algunos casos a la suerte de que el profesor le guste lo que ve y los apruebe.

Estos ejemplos se pueden comprender de una mejor manera a través de una tabla de doble entrada en el cual enfrentamos la acción de solo aprender el proceso de diseño y la acción de solo practicarlo, dándonos como resultado 4 escenarios que relacionamos a calificaciones académicas.

En el primer escenario, se puede visualizar cuando no se ha aprendido la teoría, y tampoco se ha practicado; en ese caso, lo más probable es que el alumno tenga un trabajo deplorable y tenga una nota de 05 .

En el segundo escenario, se puede ver que se ha aprendido la teoría, pero se ha practicado poco. En ese caso, es casi seguro que el alumno sepa los pasos y los implemente pero cometa muchos errores teniendo una nota de 10 .

En el tercer escenario, nos encontramos a un alumno que haya practicado muchas veces el proceso de diseño en distintos proyectos; sin embargo, la mayoría de veces fue de forma empírica y seguía lo que su criterio le intuía. En ese caso el trabajo es bueno, pero pudo haber sido mucho mejor. Nota de 15.

El cuarto escenario, es cuando el alumno conoce la teoría del proceso de diseño y la ha practicado muchas veces, dando el expertise de tener una idea previa de los pasos 
que serán más importantes de acuerdo al proyecto que le toque. Por ejemplo de recibir el proyecto de una nueva página web, reforzará la parte del brief y de los bocetos, integrando al cliente dentro del proceso, de manera tal que el resultado tenga un margen de error mínimo. Nota: 20.

\begin{tabular}{|l|c|c|}
\hline & Poco practicado & Muy practicado \\
\hline Muy estudiado & Nota 10 & Nota 20 \\
\hline Poco estudiado & Nota 05 & Nota 15 \\
\hline
\end{tabular}

Decisiones de diseño. A lo largo de todo el proceso de diseño, estamos inmersos en decisiones que como diseñadores debemos tomar responsablemente con miras a obtener un buen resultado que responda al brief. Por ejemplo, debemos decidir si esta información es útil, si esta referencia puede servir, si este color ayuda a comunicar de mejor forma el mensaje, si la tipografia puede tener un poco más de carácter, etc. Son decisiones que van encaminando el resultado del proyecto hacia un diseño que funcione, sea único y también sofisticado.

Pero tomar decisiones no es fácil. Marina Willer, diseñadora y socia de Pentagram, menciona:"cuando eres joven no tienes mucha confianza. Lo veo en muchas de las personas que trabajan conmigo. Pero si eres valiente y crees en ti mismo, realmente te ayuda a que tu trabajo se note."

Pero, ¿qué es tomar decisiones? La acción de decidir implica escoger una opción sobre otras alternativas, bajo un proceso de análisis. Lo interesante, es que no todas las personas responden de la misma forma a una problemática, esto debido a la personalidad de cada uno y a la carga del pasado. Quiénes son más reservados responderán diferente a quienes tengan una actitud más liberal.

Traducido al proceso de diseño, podemos esperar que los diseñadores afronten de diferentes formas proyectos iguales. Pero entonces, ¿cómo se puede medir el éxito de un proceso de diseño, si los resultados serán diferentes? La respuesta a esta pregunta está relaciona directamente a los objetivos del proyecto. Javier Mariscal, diseñador español lo describe muy bien:"En diseño no existe el esto es muy bonito, sino, el sí funciona o no está funcionando". Quizá el funcionamiento del diseño es la mayor de las decisiones que se debe tomar en el proceso de diseño. Esta debe ser objetiva y debe apoyarse en pruebas ácidas que se pueden realizar, sobre todo con los usuarios finales.

Sin embargo, es importante conocer algunos conceptos relacionados a la resolución de problemas y que son requisitos fundamentales para la toma de decisiones de diseño (y de todo tipo):

- Información:se debe tener la información necesaria para todas las posibles combinaciones que incluyen desde las acciones a llevar a cabo como las situaciones.

- Riesgo: una conciencia de las consecuencias a las que se hará frente al decidir ir por un camino frente al otro.

- Actitud: hacia la incertidumbre:se mide y pone a prueba la confianza del diseñador frente a lo desconocido y las probabilidades de lo que podría ocurrir.

Rudolph deHarak, diseñador gráfico estadounidense, escribió en el Aiga Journal of Graphic Deisgn, vol 5, n²,1987:"El clima donde trabaja el diseñador es muy complejo y, como en todos los campos creativos, a veces es doloroso y frustrante. El trabajo del diseñador debe satisfaces los gustos y opiniones del cliente, pero más importante aún es que debe llegar y comunicar exitosamente a las audiencias a quienes está dirigido el trabajo. Aun cuando el trabajo de diseño debería satisfacer al diseñador, frecuentemente las preferencias personales tienen escaso significado al resolver problemas de diseño".

El proceso de diseño no es algo sencillo, puede tornarse muy agobiantes en algunos casos por lo que un consejo práctico que comparto con mis estudiantes es el que Suzy Welch habla en su libro "10 minutos, 10 meses, 10 años". En este libro, Suzy nos ofrece una metodología que nos ayuda a tomar decisiones rápidas frente a escenarios de mediana complejidad. Basta con escribir las problemática sobre la cual debemos ejercer una decisión y pensar ¿cuáles serán las consecuencias en diez minutos de cada una de mis opciones?; ¿y en diez meses?; ¿y en diez años?

Aplicado hacia un horizonte de tiempo de un proyecto de diseño, yo les pido a los alumnos que se pregunten: ¿cuáles serán las consecuencias en una hora de cada una de sus opciones?; ¿y en una semana?; ¿y para final del ciclo? Este método les ayuda a pensar con una perspectiva de tiempo y no ciñéndose a la clase en curso. 
Conclusiones. En el Informe sobre el futuro del empleo 2018 del Foro Económico Mundial, se señalan cuatro competencias que serán las más importantes de cara a la cuarta revolución industrial, estas son: Creatividad, Resolución de problemas Liderazgo y gestión de personal y Pensamiento crítico.

Todas estas competencias son inherentes a los diseñadores y su capacidad para brindar soluciones. Pero estás deben estar soportadas en otras habilidades como la tomar de decisiones.

Frente a un mundo en donde la automatización de las profesiones es una amenaza, el diseñador debe continuar revalorándose no por aptitudes técnicas como usar un programa, sino por su habilidad de resolver problemas, crear mejores sistemas visuales y de comunicación e innovar para tener un mejor mundo.

Diseño y Comunicación Visual de Bruno Munari. GG 2016.

Diseño Gráfico y Comunicación de Jorge Frascara. Ediciones Infinito 1988.

Ensayos sobre Diseño, Diseñadores influyentes de la AGI. Ediciones Infinito 2001.

Señal de Diseño. Memoria de la Práctica de Ronald Shakespear. Paidós 2009.

Making Ideas Happen de Scott Belsky. Portafolio Penguin 2010.

The Grid de Matt Watkinson. Empresa Activa 2018.

10 Minutos, 10 Meses, 10 Años de Suzy Welch.Alienta Editorial.

No 1 Cuadernos. Index Book 2008.

101 Desig Methods de Vijay Kumar. John Wiley \& Sons, Inc. 2013

The Future of Jobs Report 2018 deWorld Economic Forum (http://www3 weforum.org/docs/WEF_Future_of_Jobs_2018.pdf)

https://new-ideo-com.s3.amazonaws.com/assets/files/pdfs/news/FastCo-StrategyByDesign TimBrown.pdf acaba-siendo-un-enfermo-social/

https://graffica.info/ronald-shakespear-entrevista-fotomaton/

https://concepto.de/toma-de-decisiones/

https://www.designcouncil.org.uk/sites/default/files/asset/document/ ElevenLessons_DeskResearchReport_o.pdf

https://www.designcouncil.org.uk/sites/default/files/asset/document/ ElevenLessons_Design_Council\%20(2).pdf

https://www.bbc.com/mundo/noticias-37631834

https://gestion.pe/blog/revolucion-digital/2018/02/la-mas-importante-revolucion-en-la-historia-de-la-humanidad.html/?ref $=$ ges $r$

https://hbr.org/2015/12/what-is-disruptive-innovation

https://www.designweek.co.uk/issues/25-june-1-july-2018/marina-willer-design-should-never-be-self-indulgent /

https://www.abc.es/cultura/cultural/abci-astrid-stavro-diseno-debe-prescindir-frivolo-superficial-y-modas-201903260210_noticia.html 